\title{
Pressure effects on $\alpha$-synuclein amyloid fibrils: An experimental investigation on their dissociation and reversible nature
}

\author{
Federica Piccirilli a, b, *, 1 , Nicoletta Plotegher ${ }^{c, 1}$, Francesco Spinozzi ${ }^{\mathrm{d}}$, Luigi Bubacco ${ }^{\mathrm{c}}$, \\ Paolo Mariani ${ }^{\mathrm{d}}$, Mariano Beltramini ${ }^{\mathrm{c}}$, Isabella Tessari ${ }^{\mathrm{c}}$, Valeria Militello ${ }^{\mathrm{b}}$, \\ Andrea Perucchi ${ }^{e}$, Heinz Amenitsch ${ }^{\mathrm{f}}$, Enrico Baldassarri Jr. ${ }^{\mathrm{d}, \mathrm{g}}$, Milos Steinhart ${ }^{\mathrm{h}}$, \\ Stefano Lupi ${ }^{i}$, Maria Grazia Ortore ${ }^{\mathrm{d}, * *}$ \\ a Department of Physics, University of Trieste, Trieste, Italy \\ ${ }^{\mathrm{b}}$ Department of Physics and Chemistry, University of Palermo, Palermo, Italy \\ ${ }^{c}$ Department of Biology, University of Padova, Padova, Italy \\ d Department of Life and Environmental Sciences, Marche Polytechnic University, Ancona, Italy \\ e Elettra - Sincrotrone Trieste S.C.p.A., Trieste, Italy \\ ${ }^{\mathrm{f}}$ Institute of Inorganic Chemistry, Graz University of Technology, Graz, Austria \\ ${ }^{g}$ Ny-MaSBiC (New York-Marche Structural Biology Center), Marche Polytechnic University, Ancona, Italy \\ ${ }^{\mathrm{h}}$ Institute of Macromolecular Chemistry, Prague, Czech Republic \\ ${ }^{\mathrm{i}}$ CNR IOM and Department of Physics, University of Rome La Sapienza, Rome, Italy
}

\section{A R T I C L E I N F O}

\section{Article history:}

Received 24 February 2017

Received in revised form

1 June 2017

Accepted 9 June 2017

Available online 15 June 2017

\section{Keywords:}

Amyloid

High-pressure

SAXS

FTIR

$\alpha$-synuclein

\begin{abstract}
A B S T R A C T
$\alpha$-synuclein amyloid fibrils are found in surviving neurons of Parkinson's disease affected patients, but the role they play in the disease development is still under debate. A growing number of evidences points to soluble oligomers as the major cytotoxic species, while insoluble fibrillar aggregates could even play a protection role. In this work, we investigate $\alpha$-synuclein fibrils dissociation induced at high pressure by means of Small Angle X-ray Scattering and Fourier Transform Infrared Spectroscopy. Fibrils were produced from wild type $\alpha$-synuclein and two familial mutants, A30P and A53T. Our results enlighten the different reversible nature of $\alpha$-synuclein fibrils fragmentation at high pressure and suggest water excluded volumes presence in the fibrils core. Wild type and A30P species stabilized at high pressure are highly amyloidogenic and quickly re-associate into fibrils upon decompression, while A53T species shows a partial reversibility of the process likely due to the presence of an intermediate oligomeric state stabilized at high pressure. The amyloid fibrils dissociation process is here suggested to be associated to a negative activation volume, supporting the notion that $\alpha$-synuclein fibrils are in a high-volume and high-compressibility state and hinting at the presence of a hydration-mediated activated state from which dissociation occurs.
\end{abstract}

๑) 2017 Elsevier Inc. All rights reserved.

\section{Introduction}

$\alpha$-synuclein (aSN) is a 140 amino acid protein abundant in the human brain, whose aggregation is involved in the pathogenesis of

\footnotetext{
* Corresponding author. Department of Physics, University of Trieste, Trieste, Italy.

** Corresponding author. Department of Life and Environmental Sciences, Marche Polytechnic University, Ancona, Italy.

E-mail addresses: piccirilli@iom.cnr.it (F. Piccirilli), m.g.ortore@univpm.it (M.G. Ortore).

1 The authors equally contribute to the work.
}

Parkinson's disease (PD) and other neurodegenerative disorders, known as $\alpha$-synucleinopathies. aSN belongs to the intrinsically disordered proteins (IDPs) family [1], lacking of rigid well-defined structure and it is able to adopt different conformations [2], spanning from flexible monomeric states stabilized by long range contacts, to tightly packed highly ordered aggregates, known as amyloid fibrils whose structural features have been recently determined [3]. Since this protein can fold into $\alpha$-helix when bound to lipid membranes [4] and can adopt several oligomeric or fibrillar amyloidogenic conformations if subjected to specific environmental conditions, it has been nicely defined "chameleon" by Uversky [5]. The wide interest in aSN is mainly linked to its 
implication in the etiology of PD, being in its fibrillar aggregated form one of the main constituent of Lewy bodies, proteinaceous aggregates found in the surviving neurons in PD patients. It has been recently proved that aSN can form different types of pathological aggregates, including oligomers, protofibrils and amyloid fibrils [6,7] that are believed to be responsible for the protein induced toxicity [7-9]. In particular, it seems that aSN oligomeric species are the most toxic among the protein aggregates that have been studied in cellular and animal models [10].

Nowadays it is well accepted that all proteins, if subjected to a specific environment and given a sufficient time, can be able to form amyloid fibrils [11]. In the energetic landscape, the amyloidlike state is considered the most stable state for the polypeptidic chain [12]. These aggregates show a surprising thermodynamic stability and the process leading to their formation cannot be easily reversed. The transient nature of intermediates occurring during the aSN aggregation pathway has hindered the study of their structural details and further complicated the understanding of the chemico-physical mechanisms that govern the interaction with the environment.

Differences in the structural and hydration properties of amyloid fibrils formed by wild type (WT) aSN and the two PD-linked mutants, A30P and A53T, have been reported [13,14]. More recently, it has been revealed by solid state NMR that fibrils obtained from A53T aSN in vitro show some structural variations in the core with respect to fibrils of the WT protein [15,16], while the same effect was not observed for A30P aSN fibrils [17]. Moreover, the two mutants associated with altered onset of Parkinson's disease show an accelerated aggregation kinetics in vitro [18] and differences in flexibility and solvent exposure of some amino acids [19].

Foguel et al. observed that, in spite of the thermodynamic stability shown by aSN fibrils, these highly ordered aggregates can be dissociated at high pressure [20]. de Oliveira et al. more recently studied the structural features of wild type aSN monomers resulting from high hydrostatic pressure-disturbed fibrils and investigated their capability to seed amyloid fibrils formation. Their findings support the hypothesis that the mechanism through which pressure triggers aSN amyloid fibrils to be dissociated involves hydrophobic interaction and the released monomers present altered dynamics [21]. These studies confirmed that the high pressure dissociation of aSN fibrils could provide important information concerning undetected aSN species and help to trace the road-map for fibril dissociation by exploring new approaches. Since high pressure is able to weaken hydrophobic interactions and to induce the rupture of salt bridges it has been found to be very effective for dissociating aggregates and even to induce the refolding of proteins in vitro [22].

Pressure is recognized to be a good tool for exploring and comprehending the thermodynamics of protein folding and aggregation, the structure of intermediate states as well as protein hydration and interaction properties [23-26]. The use of several pressure approaches in the last years has allowed biochemists and biophysicists to analyze the role of hydration and interactions in the formation of aggregates. For example, high-pressure effects on the structure, oligomerization state, interactions and dynamics of model proteins have been recently reported [23,25,27-31], with emphasis on hydration changes. On this basis, since high pressure induces the decrease of the total volume of the system, the destabilization processes of aSN fibrils toward states with a minor contribution of void volumes can be investigated in a controlled way by pressure.

It has been recently demonstrated that one of the main mechanisms through which pressure unfolds globular proteins is linked to the presence in the native state of cavities that are absent in the unfolded states [32]. That being so, the use of high pressure could be promising to shed light into the mechanisms behind the defects in amyloid fibrils architecture and possibly to the creation of therapies against $\alpha$-synucleinopathies [33]. However, despite the huge interest to the potential effect of high pressure on amyloid structures [34], much work is still to be done in order to clarify the changes induced in fibrils structure and to characterize the possible presence of oligomers in the pressurization of amyloid fibrils.

Given these premises, in this work we study by means of Small Angle X-ray Scattering (SAXS) and Fourier Transform Infrared Spectroscopy (FTIR) the properties of amyloid fibrils formed by WT aSN and by the two mutants A30P and A53T under high-pressure conditions. The aim is to induce fibrils dissociation at high pressure, to monitor the very first stages of their changes, and to obtain information on the differences between WT, A30P and A53T aSN aggregation mechanisms, possibly enlightening the presence of dissociation intermediates stabilized at high pressure. SAXS experiments provide the description of pressure-induced fibrils compaction at intermediate pressures, while FTIR experiments describe the relevant changes in the secondary structures obtained at the higher investigated pressures, adding further complementary information on the state of the polypeptide regions involved in the dissociation process.

\section{Methods}

\subsection{Sample preparation}

\subsubsection{Proteins purification}

WT aSN and the A30P and A53T familial mutants were expressed and purified as previously described [35]. Briefly, WT aSN, A30P and A53T, cloned into the pET-28a plasmid, were expressed into $E$. Coli strain BL21(DE3). Cells were grown at $37^{\circ} \mathrm{C}$ in $\mathrm{LB}$ broth medium to an optical density $\mathrm{OD}_{600}=0.3-0.4$ and induced with $0.1 \mathrm{mM}$ isopropyl- $\beta$-thiogalactopyranoside (IPTG) for $5 \mathrm{~h}$. Cells were collected by centrifugation and recombinant proteins were recovered from the periplasm by osmotic shock using an osmotic shock buffer. The periplasmic homogenate was boiled for 15 min and the soluble fraction containing aSN was subjected to a two-step (35\% and 55\%) ammonium sulfate precipitation. The pellet was resuspended, extensively dialyzed against $20 \mathrm{mM}$ Tris- $\mathrm{HCl} \mathrm{pH}$ 8.0, loaded into a $6 \mathrm{ml}$ Resource $\mathrm{Q}$ column (Amersham Biosciences) and eluted with a $0-500 \mathrm{mM} \mathrm{NaCl}$ gradient. After dialysis against water, aSN was lyophilised and stored as powder at $-20^{\circ} \mathrm{C}$.

\subsubsection{Fibrils preparation}

Lyophilised powders of aSN samples were re-suspended into deuterated phosphate buffered saline (PBS) at pD 7.0, filtered with a $0.22 \mu \mathrm{m}$ filter to eliminate residual small aggregates due to lyophilisation, and quantified using a UV-visible diode-array (Agilent 8453), using an extinction coefficient $\varepsilon_{276 n m}=5800 \mathrm{M}^{-1} \mathrm{~cm}^{-1}$ (1400 $\mathrm{M}^{-1} \mathrm{~cm}^{-1}$ per tyrosine residue) [36]. Fibrils were obtained aggregating the solubilized proteins at a concentration of $6 \mathrm{~g} \mathrm{~L}^{-1}$, to which $\mathrm{NaN}_{3}$ was added to a final $0.05 \%$ concentration. After 1 week at $37^{\circ} \mathrm{C}$ under $1000 \mathrm{rpm}$ shaking, fibrils were collected by pelleting for about $2 \mathrm{~h}$ and the supernatant concentration, containing the soluble residual fraction, was quantified as previously stated. The amount of protein converted to fibrils was quantified by difference, considering the amount of protein in the supernatant and the initial amount of protein in the aggregation assay. Fibrils were then resuspended in a proper volume of deuterated PBS, 
$0.05 \% \mathrm{NaN}_{3}$ to a final equivalent monomeric concentration of $10 \mathrm{~g} \mathrm{~L}^{-1}$.

\subsection{SAXS experiment}

Experiments were performed at the Austrian SAXS beamline of Elettra Synchrotron in Trieste, Italy. Measurements were carried out at $37^{\circ} \mathrm{C}$, using a high-pressure cell [37] equipped with diamond windows of $0.75 \mathrm{~mm}$ thickness each, able to cover a pressure range from $\approx 0.1$ to $\approx 1.5 \mathrm{kbar}$. The X-rays wavelength $\lambda$ was $1.54 \AA$ and the sample-detector distance was set to $2.0 \mathrm{~m}$. Hence, the modulus $Q$ of the scattering vector $(Q=4 \pi \sin \theta / \lambda, 2 \theta$ being the scattering angle) was $0.015<Q<0.4 \AA^{-1}$. Experiments were performed on mature fibrils and monomer solutions prepared as described in the previous sections at $10 \mathrm{~g} \mathrm{~L}^{-1}$ concentration. SAXS profiles were recorded on an image plate detector, and for each SAXS image the acquisition time was $5 \mathrm{~min}$. To promote the achievement of equilibrium conditions, the samples were maintained at the desired pressure value for $\simeq 5$ min before being measured. Both aSN solutions and their buffer were measured at the same pressure values, hence buffer contribution was subtracted from protein solution SAXS signal at each investigated pressure. Raw data were radially averaged and calibrated using rat-tail as reference and transmission values were measured for each investigated pressure.

\subsection{FTIR experiments}

FTIR experiments were carried out by means of a Bruker IFS66v spectrometer equipped with a Hyperion 2000 infrared microscope and a Mercury Cadmium Telluride (MCT) detector. To perform high pressure measurements we used an opposing plate screw-driven Diamond Anvil Cell equipped with natural IIa diamonds, with a culet of about $600 \mu \mathrm{m}$ and a stainless steel 316L gasket, $40 \mu \mathrm{m}$ thick. The Ruby fluorescence technique was employed for pressure calibration [38]. The way we increased and calibrated pressure, determined an uncertainty on pressure evaluation of about 0.5 kbar. The pressure calibration was performed directly on the microscope through the use of a home-made fluorescence system [29]. Measurements were carried out in transmission mode, in the frequency range between 400 and $4000 \mathrm{~cm}^{-1}$, under continuous purging in $\mathrm{N}_{2}$ dry atmosphere. Typically 256 interferograms were co-added at $2 \mathrm{~cm}^{-1}$ resolution. Spectra were baseline corrected and normalized according to [29] and data analysis was performed by Opus Pro 6.5 Software Package (Bruker).

\section{Results and discussion}

SAXS and FTIR experiments were performed on WT aSN, mutants (A30P and A53T) monomers and on their fibrillar products. Sample solutions were subjected to increasing pressures, and their effects and the reversibility of the process were tested for each kind of species. Sample solutions of aSN monomers, which are expected to be in an intrinsically disordered conformation [39], do not evidence relevant changes due to the high-pressure treatment (data reported in Supplementary Materials). On the other side, SAXS and FTIR experimental data on aSN fibrils result to be affected by the pressure treatment and provide useful information on the ranges of pressure that cause a change in the fibril structure as well as on the reversibility of the processes.

\subsection{SAXS}

SAXS curves related to WT, A30P and A53T starting states, prior to fibrillation and prior to adding pressure, are reported in Fig. 1. According to the developed protocol [35], aSN starting states are expected to be mainly monomeric. Since a disordered conformation is foreseen for WT and for each familial mutant, scattering data for starting states are analysed considering the fitting macroscopic differential scattering cross section $d \Sigma / d \Omega(Q)$ written for wormlike species in solution:

$$
\begin{aligned}
\left.\frac{d \Sigma}{d \Omega}\right|_{w l k}(Q)= & \frac{c N_{A}}{M_{1} N_{a, w l k}} r_{e}^{2} 4 \pi^{2} L_{\mathrm{aSN}}^{2}\left[\left(\rho_{1}-\rho_{0}\right) R_{\mathrm{aSN}}^{2} \frac{J_{1}\left(Q R_{\mathrm{aSN}}\right)}{Q R_{\mathrm{aSN}}}\right. \\
& \left.+\left(\rho_{\mathrm{s}}-\rho_{0}\right) \times\left(R_{\mathrm{aSN}}+\delta\right)^{2} \frac{J_{1}\left(Q\left(R_{\mathrm{aSN}}+\delta\right)\right)}{Q\left(R_{\mathrm{aSN}}+\delta\right)}\right]^{2} P_{\mathrm{ped}}(Q)
\end{aligned}
$$
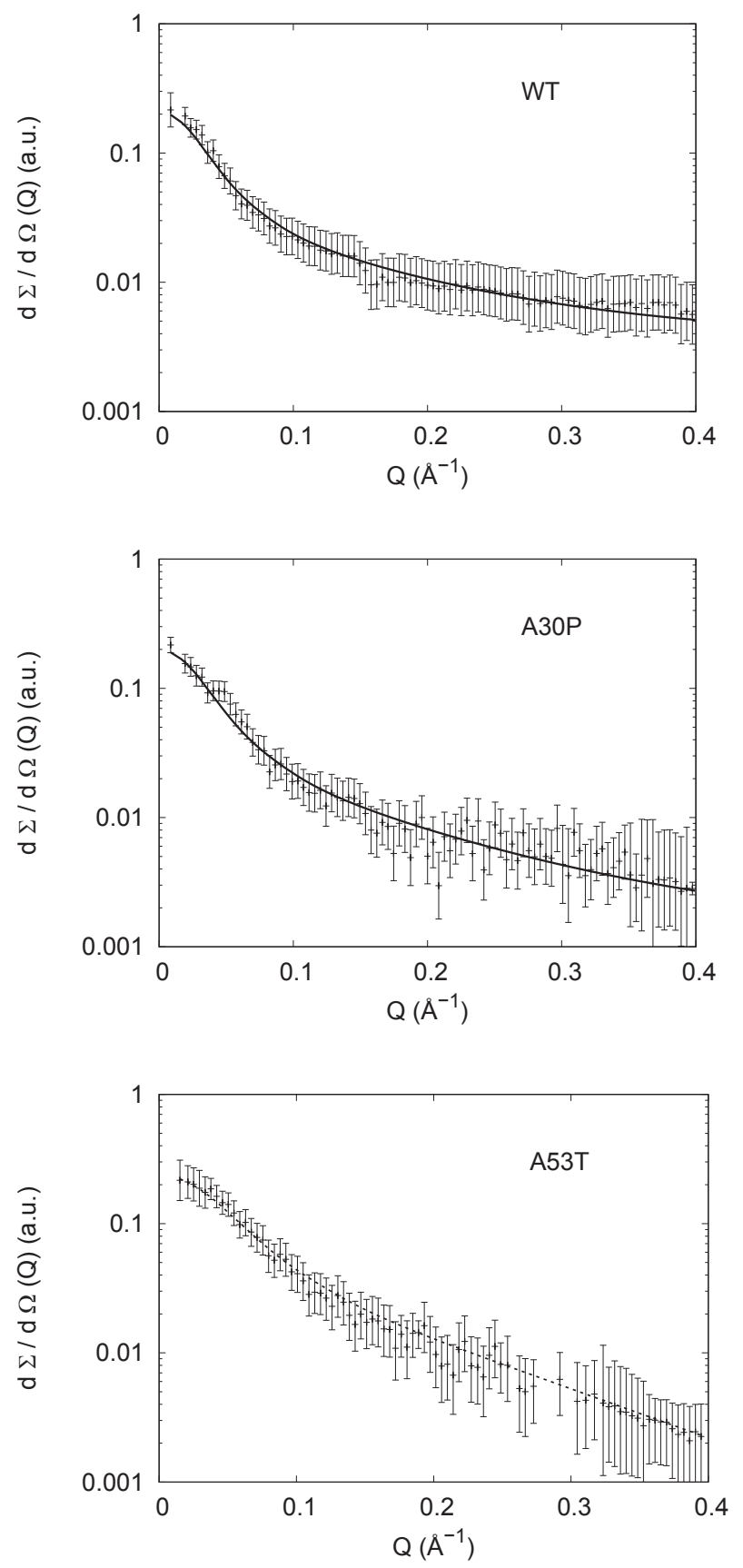

Fig. 1. Experimental SAXS curves of in-solution WT, A30P and A53T aSN starting conditions as monomers, as in the legend. All samples are at pressures $p=0.1 \mathrm{kbar}$. 
where $J_{1}(x)$ is the first order Bessel function, $L_{\mathrm{aSN}}, R_{\mathrm{aSN}}$ and $\rho_{1}$ are the contour length, the cross-sectional radius and the uniform electron density of the protein chain, respectively, and $\delta$ and $\rho_{s}$ are the thickness and the electron density of the surrounding shell, which takes into account the heterogeneous nature of the proteinsolvent interface (e.g., the contribution of the water in the hydration shell and any effect due to the protein side chains). The worm-like form factor $P_{\text {ped }}(Q)$ contains the statistical segment (Kuhn) length $b$, representing the separation between two adjacent rigid scattering domains (note that the number of statistical segments $n_{b}$ can be derived by $n_{b}=L_{\mathrm{aSN}} / b$ [40]). The aggregation number $N_{a, w l k}$ is the number of aSN monomers that form each flexible chain and is determined by the ratio $\pi R_{\mathrm{aSN}}^{2} L_{\mathrm{aSN}} / V_{1}$, where $V_{1}$ is the aSN monomer volume. Experimental SAXS curves fitted by means of GENFIT software [41] resulted to be coherent with the hypothesis of monomeric disordered peptides in solution $\left(N_{a, w l k} \simeq 1.05\right)$, and the resulting parameters are reported in the Supplementary Materials.

The effect of pressure on amyloid fibrils is monitored as a function of pressure by measuring samples after a fixed equilibration time and as a function of time by following the evolution of samples state toward the equilibrium. In fact high pressure effects on amyloid fibrils can increase at increasing times as revealed in a previous study on transthyretin and aSN fibrils [20]. Hence, since a kinetic effect of pressure on amyloid fibrils can be expected, we have also monitored the modification induced by the highest investigated pressure at different times, until $4 \mathrm{~h}$ after triggering the compression process, for each species of aSN fibrils.

On the basis of previous literature results [42-46], SAXS curves of the three species of aSN fibrils at ambient pressure were successfully analysed considering the simple model of right cylinders. However, since a soluble fraction of disordered aggregates cannot be completely avoided, SAXS curves of aSN fibrils were fitted considering the simultaneous presence of cylinders and of disordered (worm-like) species.

It has to be stressed that SAXS curves obtained with the high pressure cell equipped with diamond windows show a signal-tonoise ratio lower than the one usually achieved by standard synchrotron SAXS sample cells, and a simultaneous exact protein concentration measurement by UV detector, as performed in recent SAXS studies on aSN [47], is not possible in our set-up conditions. Hence, the differences between WT and A30P aSN fibrils detected by Nielsen et al. [48], suggesting increased $\beta$ sheet pairing distances in A30P fibrils and their more compact interfibrillar arrangement, cannot be evidenced in our experimental set-up, neither the use of a single helix form factor newly adopted to analyze lysozyme amyloid aggregates [49] is suitable for the analysis of our SAXS curves. In agreement with Atomic Force Microscopy results [50], we have considered that the cylinder radius could be polydisperse, and fibril electron density could be lower from the one of globular protein, due to the hypothesis of the presence of internal water domains [51,52]. Due to the moderately low sample concentration, to the high aggregation number of amyloid fibrils that determines a low number density of scattering particles in solution, to the lowest available $Q$ value and to the absence of interaction peaks in our SAXS curves, the interactions between fibrils have been neglected. Hence the effective structure factor $S(Q)$ [53] has been considered equal to unity, in agreement with previous similar experimental conditions [54-56]. The form factor of polydispersed radius cylinders [57], with uniform electron density $\rho_{e}$, average radius $R_{C}$ with dispersion $\xi_{R_{c}}$ and length $L$ we have used to represent amyloid fibrils is:

$$
\begin{aligned}
& \left.\frac{d \Sigma}{d \Omega}\right|_{c y l}(Q)=\frac{c N_{A}}{M_{1}} \frac{\pi L V_{1}}{R_{c}^{2}\left(1+\xi_{R_{c}}^{2}\right)} r_{e}^{2}\left(\rho_{1}-\rho_{0}\right)\left(\rho_{e}-\rho_{0}\right) \\
& \quad \times \int_{0}^{\infty} d R f(R) \int_{0}^{\pi / 2} d \beta \sin \beta \\
& \quad \times\left\{2 R^{2} \frac{\sin \left(\frac{1}{2} Q L \cos \beta\right)}{\frac{1}{2} Q L \cos \beta} \frac{J_{1}(Q R \sin \beta)}{Q R \sin \beta}\right\}^{2}
\end{aligned}
$$

where the bulk solvent electron density $\rho_{0}$ has been let to change with pressure on the basis of literature values at zero pressure of water molecular volume, $v^{\circ}$, water compressibility, $\beta^{\circ}$, and water isothermal bulk modulus, $\eta[58,59], \rho_{0}=n_{\text {wat }} /\left\{v^{\circ}\left[1-\beta^{\circ} p /\{1+\right.\right.$ $\left.\left.\left.(\eta+1) \beta^{\circ} p / 2\right\}\right]\right\}, n_{\text {wat }}$ being the number of electrons in the water molecule. The electron density of aSN monomer, $\rho_{1}$, has been expressed as a function of pressure by considering its value at zero pressure calculated on the basis of amino acid composition, $\rho_{1}^{\circ}$, and the standard value of protein compressibility $\beta_{1}$ [60], $\rho_{1}=\rho_{1}^{\circ} \exp \left(\beta_{1} p\right)$. The normalized $\log$-normal function $f(R)$ has been selected to describe the probability density distribution of the cylinder radius $R$ [41].

aSN fibrils in solution are expected to be altered and dissociated by high pressure, hence in each experimental condition the simultaneous presence of fibrils and soluble disordered aggregated is considered as:

$\frac{d \Sigma}{d \Omega}(Q)=\left.x_{c y l} \frac{d \Sigma}{d \Omega}\right|_{c y l}(Q)+\left.x_{w l k} \frac{d \Sigma}{d \Omega}\right|_{w l k}(Q)$

where $x_{c y l}$ and $x_{w l k}$ are the fraction of monomer that forms the fibrils and the oligomeric soluble states, respectively $\left(x_{c y l}+x_{w l k}=1\right)$ [61]. The theoretical curves suitably fit the experimental SAXS data, as it can be appreciated in Fig. 2. Further details on SAXS data analysis, concerning the mathematical steps leading to Equation (2) and the set of fitting parameters, are reported in Supplementary Materials. The most relevant fitting parameters are plotted in Fig. 3. Results show that high pressures available at the SAXS beamline until $1.6 \mathrm{kbar}$ - can subtly modify fibrils' sizes and density features, maintaining their overall morphological structure, and induce the fragmentation into soluble disordered aSN oligomers. The possible simultaneous presence of different oligomeric species, as recently detected and described in different experimental conditions by SAXS experiments $[9,47,62]$, can be pointed up from our SAXS curves. Both wild type and familial mutants of aSN amyloid fibrils show that at increasing pressures the fraction of soluble disordered states is increasing. However, it has to be noticed that in this kind of experiment, SAXS technique resolution is not able to distinguish between different oligomers produced by high pressure, and the resulting parameters are affected by meaningful error bars. On the other side, each solution of aSN amyloid fibril we investigated presents similar features at increasing pressures. The average radius $R_{c}$ of WT, A30P and A53T aSN fibrils decreases with pressure, whereas the fibril electron density $\rho_{e}$ is found to be always lower than the nominal electron density of protein $\left(\rho_{1}\right)$ and increases with pressure. Also, the rate of increase of fibrils' electron density with pressure is slightly larger than the one it could be expected (see Fig. 3). In fact, considering the standard protein compressibility values, the electron density of fibrils should follow the continuous line shown in Fig. 3. The difference between the expected behavior and the one resulting from SAXS data analysis can be ascribed to 

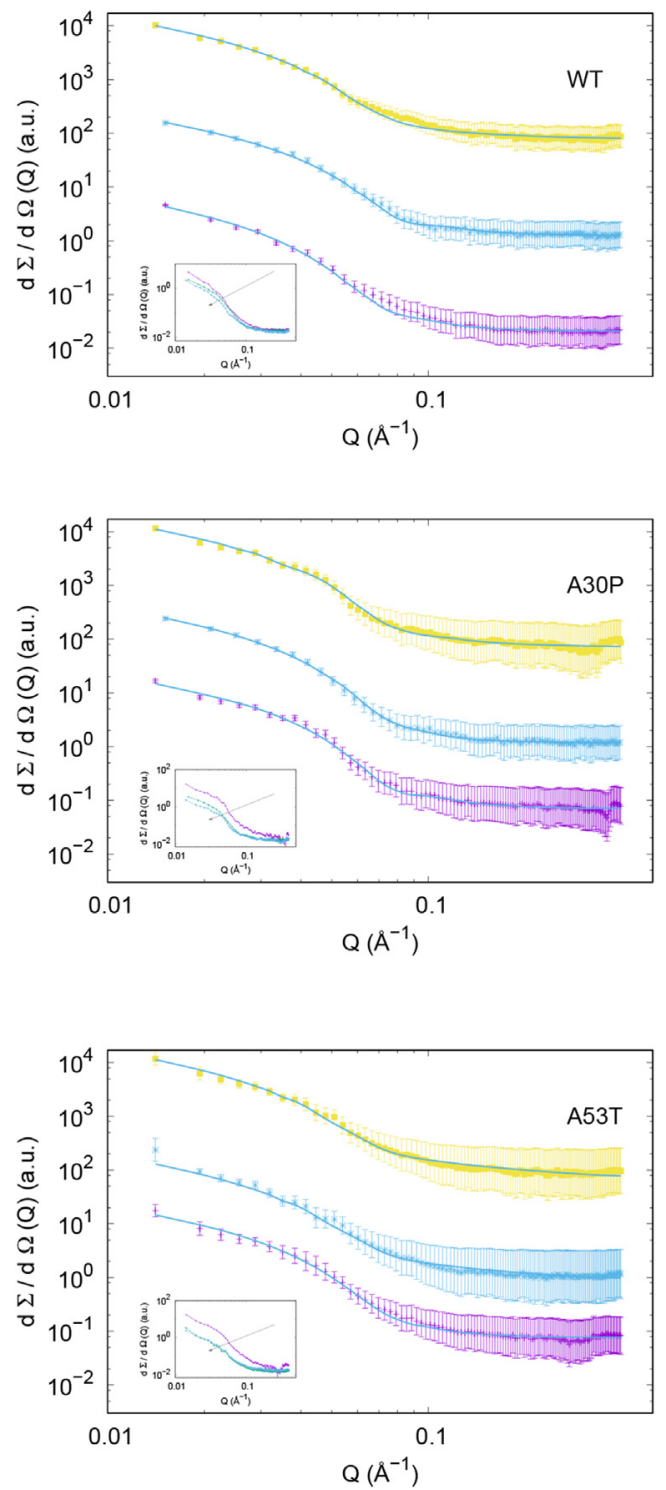

Fig. 2. Experimental SAXS curves of in-solution WT, A30P and A53T aSN fibrils, as in the legend, at different pressure values. From bottom to top, SAXS curves refer to $p=$ $0.1 \mathrm{kbar}$ (violet), to $p=0.8 \mathrm{kbar}$ (cyan), and to $p=1.6 \mathrm{kbar}$ (yellow). Curves are scaled for clarity for a factor $f(f=70)$. Solid lines are the best fits obtained considering a combination of cylinders resembling amyloid fibrils and disordered soluble aggregates, as described in the text. Insets report the same experimental SAXS curves without scaling, and the arrows indicate the trend as pressure increases. (For interpretation of the references to colour in this figure legend, the reader is referred to the web version of this article.)

the evidence that, similarly to folded proteins, fibrillar aggregates have packing defects that modify their answer to pressure. Hence, the decrease of the average fibril radius with pressure and the simultaneous increase of their electron densities, can be considered the signature of a gradual disappearing of internal cavities, electrostriction of broken electrostatic interactions and additional hydration [63]. These results seem to confirm the existence of packing defects in the fibril core and the participation of hydrophobic interactions in their structure that make them responsive to moderate pressures. On the other side, the fraction of monomers inside amyloid fibrils according to SAXS results decreases in favor of soluble disordered species. Hence, SAXS results can confirm a partial dissociation suggested in a previous Light Scattering study [20], where a wider pressure range has been explored.
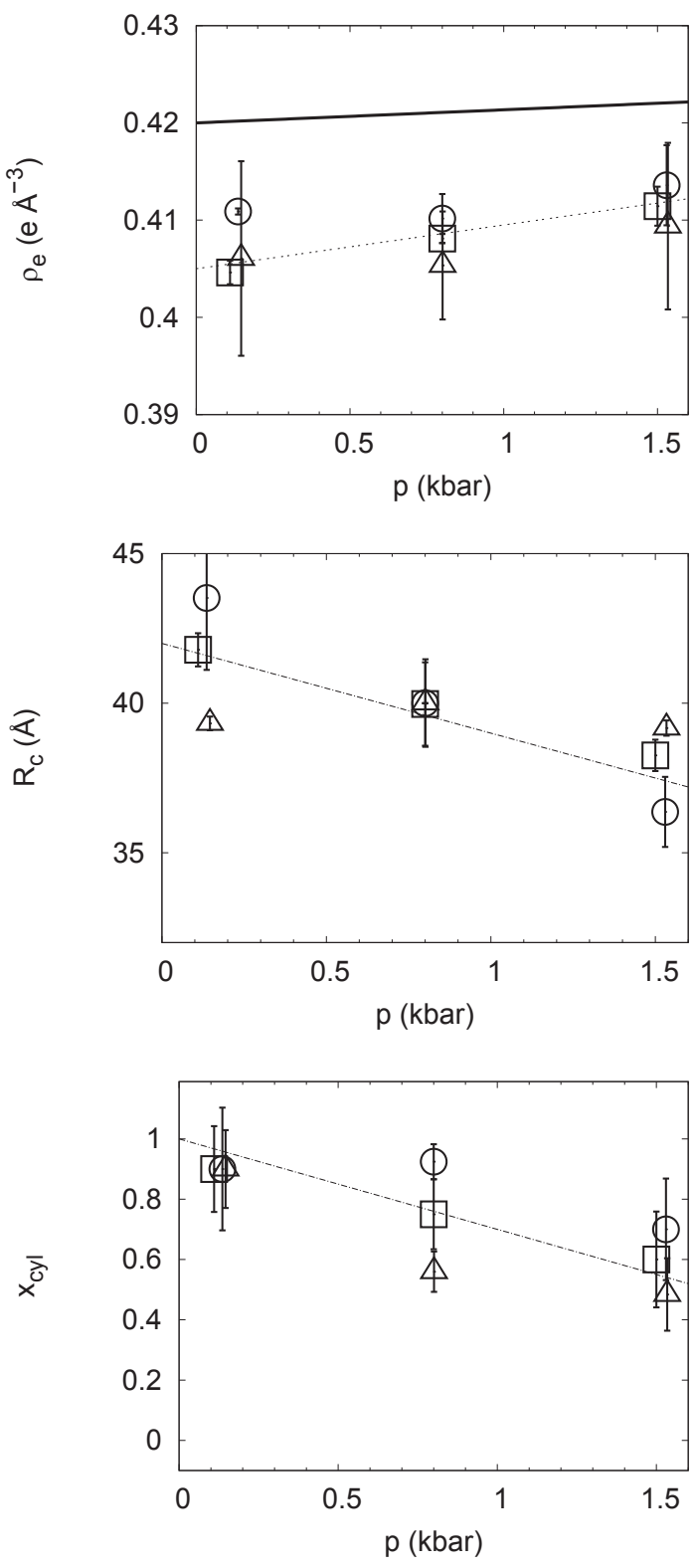

Fig. 3. Fitting parameters together with their error bars resulting from SAXS analysis: squares are for WT, circles for A30P, and triangles for A53T. Top: amyloid fibrils electron densities, where the continuous line represents the electron density change expected considering standard protein electron density and compressibility, while dashed line is a guide to the eye. Middle: cylinders average radius as function of pressure (the line is a guide to the eyes). Bottom: fraction of monomer in fibrillar species at increasing pressures (the line is a guide to the eyes).

Albeit fibril preparation and concentration adopted in our experiments are the same as those used by Foguel et al. [20], we consider that little differences in the results can derive from differences in sample features in terms of homogeneity and pressure resistance, maybe related to sample aging, to sample holder features, and/or to the deuterated solvent, as it was found in the case of hen egg white lysozyme fibrils [49]. In brief, SAXS experiments confirm the existence of packing defects in aSN fibrils, the possible presence of soluble disordered species in solution, and suggest that the very first-order effects of the high pressure treatment to mature amyloid fibrils are related to hydration changes. 


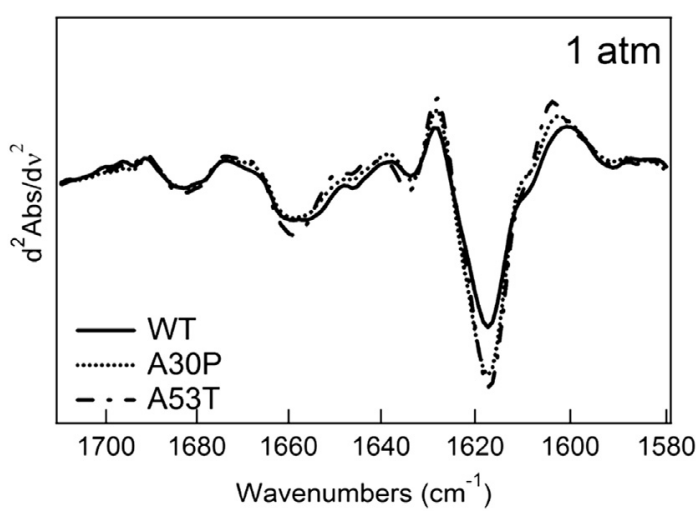

Fig. 4. Second derivative of the amide $I^{\prime}$ absorption band of WT and mutant aSN amyloid fibrils at ambient pressure.

\subsection{FTIR}

FTIR spectra have been recorded on fibrils in solution formed by WT aSN and by A30P and A53T mutants at increasing pressures until 5.5 kbar. Differently from SAXS high pressure cell, the equipment adopted for FTIR experiments allows to investigate a much wider pressure range, albeit with less accuracy in the low pressure range, $p<1.5 \mathrm{kbar}$. Fig. 4 shows the absorption band in the amide $I^{\prime}$ region for the three samples at ambient pressure (expressed in the form of $2^{\text {nd }}$ derivative) and during the first compression cycles. The amide I band, referred to as amide $\mathrm{I}^{\prime}$ in deuterated environment, placed between 1580 and $1710 \mathrm{~cm}^{-1}$, is mainly composed by $\mathrm{C}=\mathrm{O}$ stretch (building up the $80 \%$ of the band) and $\mathrm{N}-\mathrm{H}(\mathrm{N}-\mathrm{D})$ band absorption and is commonly used in the determination of the secondary structure of proteins due to the high sensitivity of the $\mathrm{C}=\mathrm{O}$ stretching to its own $\mathrm{H}$-bond state. Each secondary structure segment gives rise to a distinct $C=0$ absorption feature $[64,65]$. No clear differences have been previously reported between fibrils produced by WT and A30P and A53T mutant forms of aSN [14] by FTIR spectra: Fig. 4 indeed confirms the similarity between the three fibrillar species [66].

High-pressure FTIR spectra of the investigated fibrils during the compression cycles are shown in Fig. 5. A qualitative view already evidences that hydrostatic pressures higher than 2.0 kbar noticeably modify FTIR fingerprints of the three fibrillar species. At the lowest applied pressures, the spectra show the typical shape of misfolded aggregates, featuring a narrow peak at about $1617 \mathrm{~cm}^{-1}$ with a shoulder at around $1660 \mathrm{~cm}^{-1}$. The qualitative structural composition of the fibrillar aggregates, evaluated from the position of the peaks in the $2^{\text {nd }}$ derivative spectra at the lowest applied pressures, is reported in Table 1. Fig. 5 also shows that by increasing pressure the FTIR signal in the $1630-1680 \mathrm{~cm}^{-1}$ region, where random coils and intramolecular structures are known to absorb, increases whereas the signal at around $1617 \mathrm{~cm}^{-1}$, assigned to intermolecular $\beta$-sheets, decreases. The amide $\mathrm{I}^{\prime}$ bands observed at the highest applied pressure, are peaked at around $1645 \mathrm{~cm}^{-1}$, and appear to be quite symmetric with a slight shoulder at around $1617 \mathrm{~cm}^{-1}$. These absorption bands result not very different from the one corresponding to native aSN, as reported by Uversky et al. [67], suggesting a structural randomization of the proteins at the highest pressures. We notice that the complete suppression of the peak centered at about $1617 \mathrm{~cm}^{-1}$ (see Fig. 5), corresponding to the dissociation of most intermolecular $\beta$-sheets in the fibrillar aggregate, occurs at the highest available pressure. This result could be attributed to a possible high spatial inhomogeneity of the local compressibility of the fibrillar aggregate that leads, at high
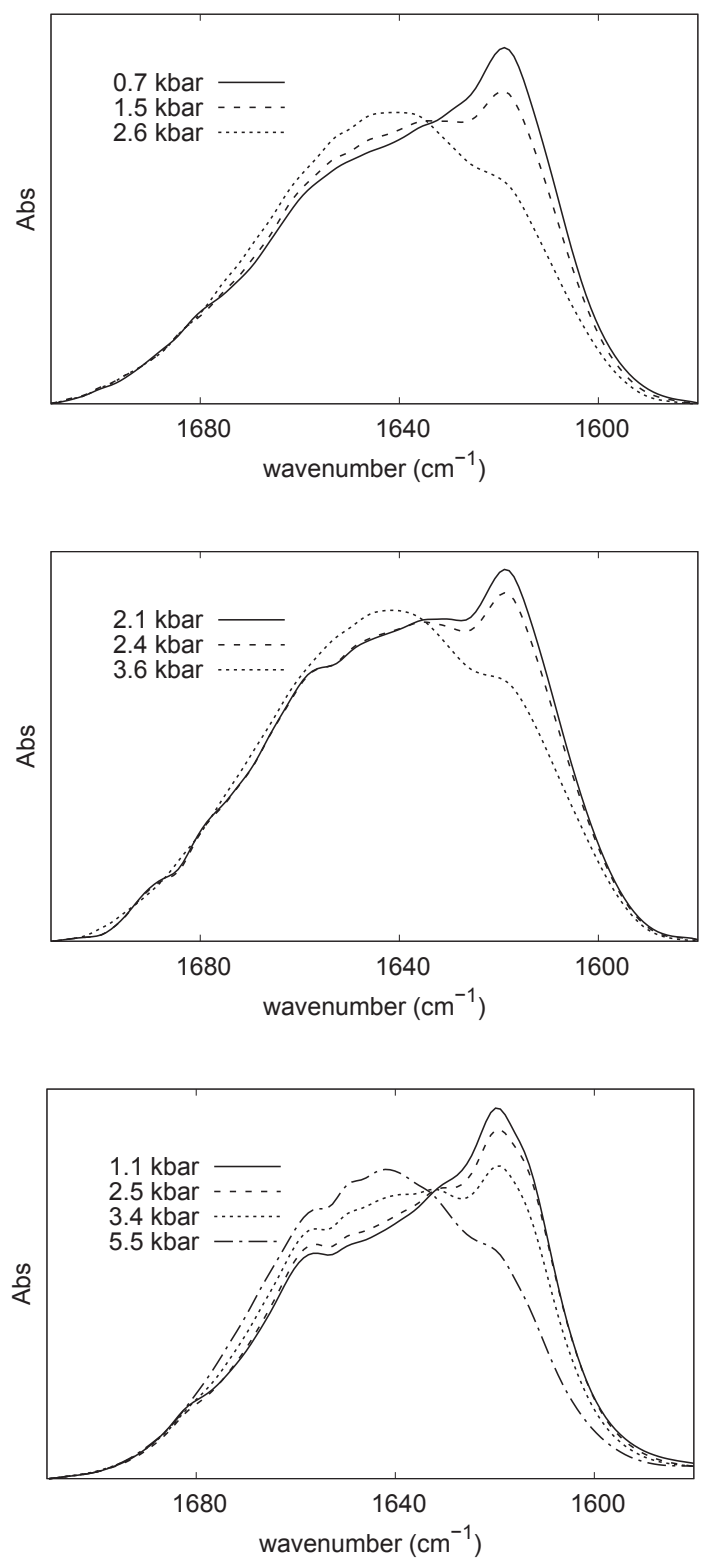

Fig. 5. Amide $\mathrm{I}^{\prime}$ absorption band of $\mathrm{WT}$ (top), A30P (middle) and A53T (bottom) aSN amyloid fibrils during the compression cycle, as reported in the legend (uncertainty in pressure calibration is $\simeq 0.5 \mathrm{kbar}$ ).

pressure, to a gradual shift of the equilibrium towards aSN monomeric states.

In order to quantitatively compare the sensitiveness of the three aSN fibrillar species to pressure, we have calculated the ratios between the intensity of the signal coming from intermolecular $\beta$ sheet at around $1617 \mathrm{~cm}^{-1}, I_{\beta}$, and the one at the isosbestic point

Table 1

Secondary structure assignment for aSN fibrils at the lowest pressure applied.

\begin{tabular}{llll}
\hline Structural component & $\begin{array}{l}\text { WT (0.7 kbar) } \\
\text { Wavenumber } \\
\left(\mathrm{cm}^{-1}\right)\end{array}$ & $\begin{array}{l}\text { A53T (1.1 kbar) } \\
\text { Wavenumber } \\
\left(\mathrm{cm}^{-1}\right)\end{array}$ & $\begin{array}{l}\text { A30P }(2.1 \mathrm{kbar}) \\
\text { Wavenumber } \\
\left(\mathrm{cm}^{-1}\right)\end{array}$ \\
\hline aggregated strands & 1692 & - & 1692 \\
$\beta$-sheet (intermol), turn & 1679 & 1677 & 1683 \\
helix, loops & 1659 & 1660 & 1660 \\
random coil & - & 1645 & 1646 \\
$\beta$-sheet (intramol) & 1636 & 1635 & 1635 \\
$\beta$-sheet (intermol) & 1618 & 1617 & 1618 \\
\hline
\end{tabular}




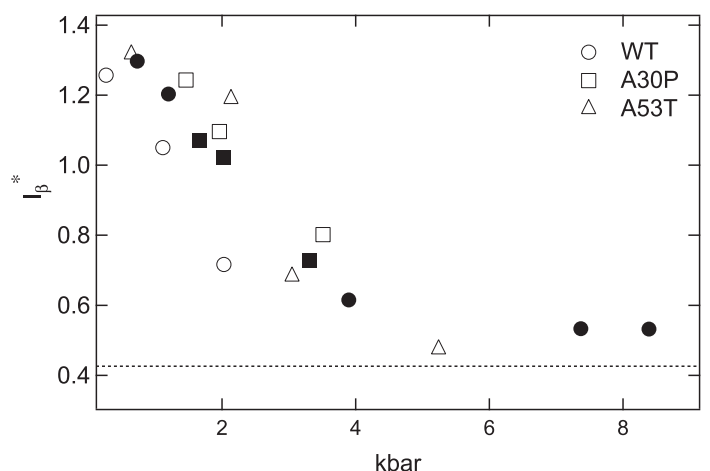

Fig. 6. Ratios $I_{\beta}^{*}=I_{\beta} / I_{\text {iso }}$ (where $I_{\beta}$ refers to $1617 \mathrm{~cm}^{-1}$ and $I_{\text {iso }}$ to $1630 \mathrm{~cm}^{-1}$ ), as a function of pressure. Empty and solid symbols refer to the first and the second compression cycles, respectively. The dashed line represents the value of $I_{\beta} *$ found for the native form of WT aSN. Symbols as in the legend.

drawn by high pressure bands (at around $1630 \mathrm{~cm}^{-1}$ ), $I_{\text {iso }}$. The value $I_{\beta}^{*}=I_{\beta} / I_{\text {iso }}$, considered as representative of the aggregation degree of the sample, is reported as a function of pressure for the three aSN species in Fig. 6. Just small differences among WT, A30P and A53T aSN can be appreciated, suggesting that the three species are almost equally affected by high pressure. Of note, at pressures around $5.0 \mathrm{kbar}$, the structural changes induced by high pressure are found to be completed.

\subsubsection{Kinetics analysis of FTIR and SAXS data}

Further information concerning aSN fibrils species response to high pressure can be provided by exploring the kinetics of the amide $I^{\prime}$ bands at fixed pressures $[20,68,69]$. In fact, time evolution of the FTIR absorption intensity $I_{\beta}(t)$ at $1619 \mathrm{~cm}^{-1}$, considered as an amyloid fingerprint, is analysed for the three investigated samples at different pressure values. Both SAXS signals and FTIR curves can be successfully fitted by a simple exponential decay. FTIR data could even be fitted by adopting a more complex model, in which two main time decays can be observed, in agreement with Oliveria results [21]. However, because it was not possible to achieve confident results by adopting bi-exponential fit for all the samples because experimental times did not always match the temporal characteristics of the kinetics, we chose to adopt the simplest single exponential decay. The following equation was used to fit the data:

$I_{\beta}(t)=I_{\beta}(\infty)+\left[I_{\beta}(0)-I_{\beta}(\infty)\right] \exp \left(-k_{\text {dis }} t\right)$

where $I_{\beta}(0)$ is the initial absorption peak intensity, $I_{\beta}(\infty)$ is the intensity at infinite time (i.e., when the signal did not change anymore) and $k_{\text {dis }}$ is the estimated kinetic constant for dissociation [68]. At each pressure the change in the renormalized absorption peak intensity at $1619 \mathrm{~cm}^{-1}$ is plotted against time, together with the fitting curves (Fig. 7). The dissociation constants are reported in Table 2 in units of $s^{-1}$, in order to be easily compared to literature. In particular, our resulting constants are of the same size of those derived by Hasegawa et al. [70] for dissociation of amyloid- $\beta$ fibrils. Results relative to WT and A30P kinetics show that the kinetic constants $k_{\text {dis }}$ increase with pressure. This evidence suggests that the amyloid fiber dissociation is guided by a negative change of the protein volume. The negative activation volume supports the notion that the aSN amyloid fibrils are in a high-volume and highcompressibility state, hinting at the involvement in the dissociation process of a hydration-mediated activated state of the fibril [68]. Interestingly, while the evaluated rates for WT and A30P mutant are mutually consistent, the dissociation of A53T amyloids appears slower.

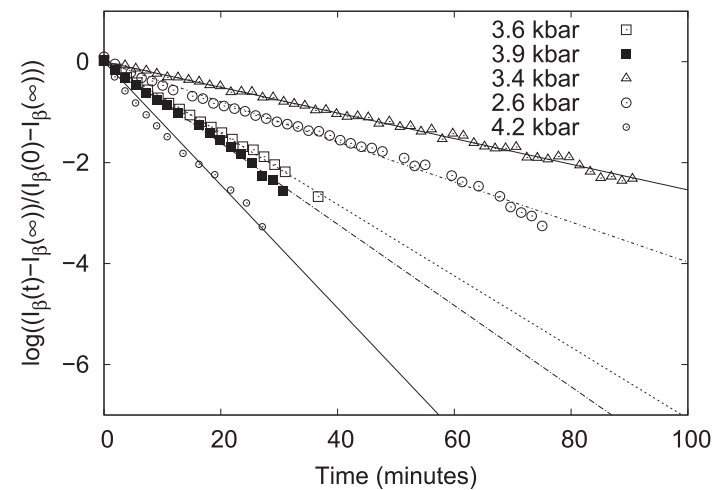

Fig. 7. Plot of logarithms of the normalized $\beta$-sheet signals, calculated as described in FTIR Section, as a function of time at the fixed pressure reported in the legend. Circles refer to WT, squares to A30P and triangles to A53T aSN species. Empty symbols refer to the first pressure cycle, while full symbols correspond to the second cycle of compression.

Although SAXS and FTIR signals provide different structural information, on overall molecular dimensions and on amyloid $\beta$ structures, respectively, it is stimulating to compare the kinetic response, at least in terms of the dissociation constants. Hence, we have determined the kinetics of SAXS signals at the highest investigated pressure. In detail, the ratios between SAXS intensities at the lowest $Q$ experimental values, renormalized according to the previous equation adopted for FTIR intensities (considering $I(Q \simeq 0, t)$ in place of $I_{\beta}(t)$ in Eq. (4)) are reported in Fig. 8, considering the intensity as soon as the pressure value is obtained, and the intensity at infinite time, just like it has been done with FTIR signal at $1619 \mathrm{~cm}^{-1}$. All the resulting kinetic constants are reported in Table 2.

First of all, it is noticeable that both SAXS and FTIR signals present a single step kinetic that dominates the dissociation processes, at least in the explored time scale and pressure range. Secondly, $k_{\mathrm{dis}}$ values obtained by the two different techniques are the same in order of size: this result could suggest that fibril dissociation proceeds in the same time scale both for fibrils dissociation and for $\beta$ sheets structural changes. Also, dissociation constants are bigger for A30P species in respect to WT, both for SAXS and for FTIR results. Hence, these results reveal a primary structure dependence of dissociation rates not enlightened before, being mutant fibrils kinetics faster than the one observed for WT. This evidence is in accordance with the results of Foguel et al. [20] obtained by light scattering experiments. Considering that the two techniques here employed provide different structural information on overall molecular dimensions and on amyloid $\beta$ structures, the analysis of

Table 2

Dissociation rate constants $k_{\text {dis }}$ obtained at constant pressures ranging from 1.6 up to 4.2 kbar from linear fitting the FTIR data reported in Fig. 7 and the SAXS data reported in Fig. 8. Bold numbers refer to SAXS data analysis, while the others to FTIR data analysis.

\begin{tabular}{llll}
\hline & \multicolumn{3}{c}{ WT } \\
\hline$p($ kbar $)$ & $\mathbf{1 . 6}$ & 2.6 & 4.2 \\
$k_{\text {dis }}\left(10^{-4} \mathrm{~s}^{-1}\right)$ & $\mathbf{5 . 2 6} \pm \mathbf{0 . 0 6}$ & $6.62 \pm 0.06$ & $20.3 \pm 0.5$ \\
\hline & \multicolumn{3}{c}{ A30P } \\
\hline$p($ kbar $)$ & $\mathbf{1 . 6}$ & 3.6 & 3.9 \\
$k_{\text {dis }}\left(10^{-4} \mathrm{~s}^{-1}\right)$ & $\mathbf{1 3 . 2} \pm \mathbf{0 . 3}$ & $11.78 \pm 0.06$ & $13.4 \pm 0.1$ \\
\hline & & A53T & \\
\hline$p($ kbar $)$ & $\mathbf{1 . 6}$ & 3.4 & \\
$k_{\text {dis }}\left(10^{-4} \mathrm{~s}^{-1}\right)$ & $\mathbf{2 1 . 3} \pm \mathbf{0 . 3}$ & $4.23 \pm 0.02$ & \\
\hline
\end{tabular}




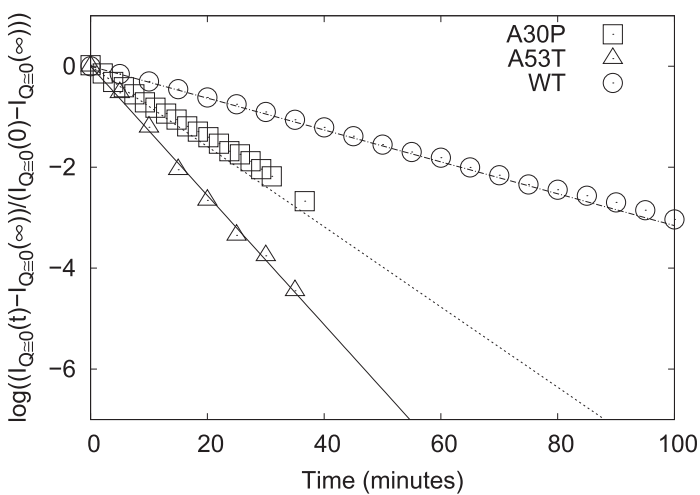

Fig. 8. Plot of logarithms of the extrapolated SAXS intensity at $Q=0$, normalized as described in the text, as a function of time at $p=1.6 \mathrm{kbar}$. aSN species corresponding to symbols are reported in the legend.

dissociation kinetics suggests that a superposition of different processes, occurring on different length scales and high pressure ranges, can compete during high pressure dissociation of aSN fibrils.

Concerning the reversibility of the process induced upon decompression, amide I' bands measured before and after the high pressure treatment, reported in Fig. 9, evidences that WT and A30P aSN fibrils show a rather clear restore of the initial signal, suggesting a total reversibility of the processes triggered by high pressure. On the contrary, A53T mutant shows only a partial rebuilding of the starting signal, indicating an incomplete protein re-aggregation after pressure release.

On the other side, the kinetic response of A53T fibrils to high pressure treatment is dissimilar to the one observed for WT and A30P aSN fibrils. In order to explain this different behavior of A53T fibrils in comparison with the ones from WT and A30P aSN, we report in Fig. 10 the $2^{\text {nd }}$-derivative spectra of the amide I' FTIR band for the three species stabilized upon compression. In the case of WT and A30P fibrils, we do not observe any $\beta$-sheet component other than a residual fibrillar one absorbing at about $1619 \mathrm{~cm}^{-1}$ Conversely, the A53T sample shows a band at $1628 \mathrm{~cm}^{-1}$ attributed to peptide chains with weakly coupled $\beta$-sheets [30]. The presence of this absorption moiety could possibly reflect the presence of intermediate, possibly oligomeric $\beta$-sheet rich species.

Hence, the stabilization of these structures upon compression could thus furnish a possible explanation for the slower dissociation rate observed for A53T fibrils. The criterion according to which the decrease of the signal at about $1619 \mathrm{~cm}^{-1}$ provides information about the kinetics of dissociation relies in fact on the assumption that the absorption intensity at that frequency is quantitatively representative of the content of $\beta$-sheet present in the sample. This condition is not satisfied if there is more than one contribution from species composing the sample absorption spectrum in the region around $1619 \mathrm{~cm}^{-1}$. The presence of a stable intermediate, as product of high pressure dissociation, detectable for A53T fibrils and not present in WT and A30P fibrils spectra, could thus explain the slower dissociation rate observed for A53T mutant aggregates. This suggests that A53T mutant could be dissociated by high pressure in oligomeric units.

\section{Conclusion}

Our experimental results provides further perspectives about the use of high pressure on amyloids species. Both SAXS and FTIR data detect small structural differences between WT, A30P and A53T aSN fibrils response to high pressure. In particular, the
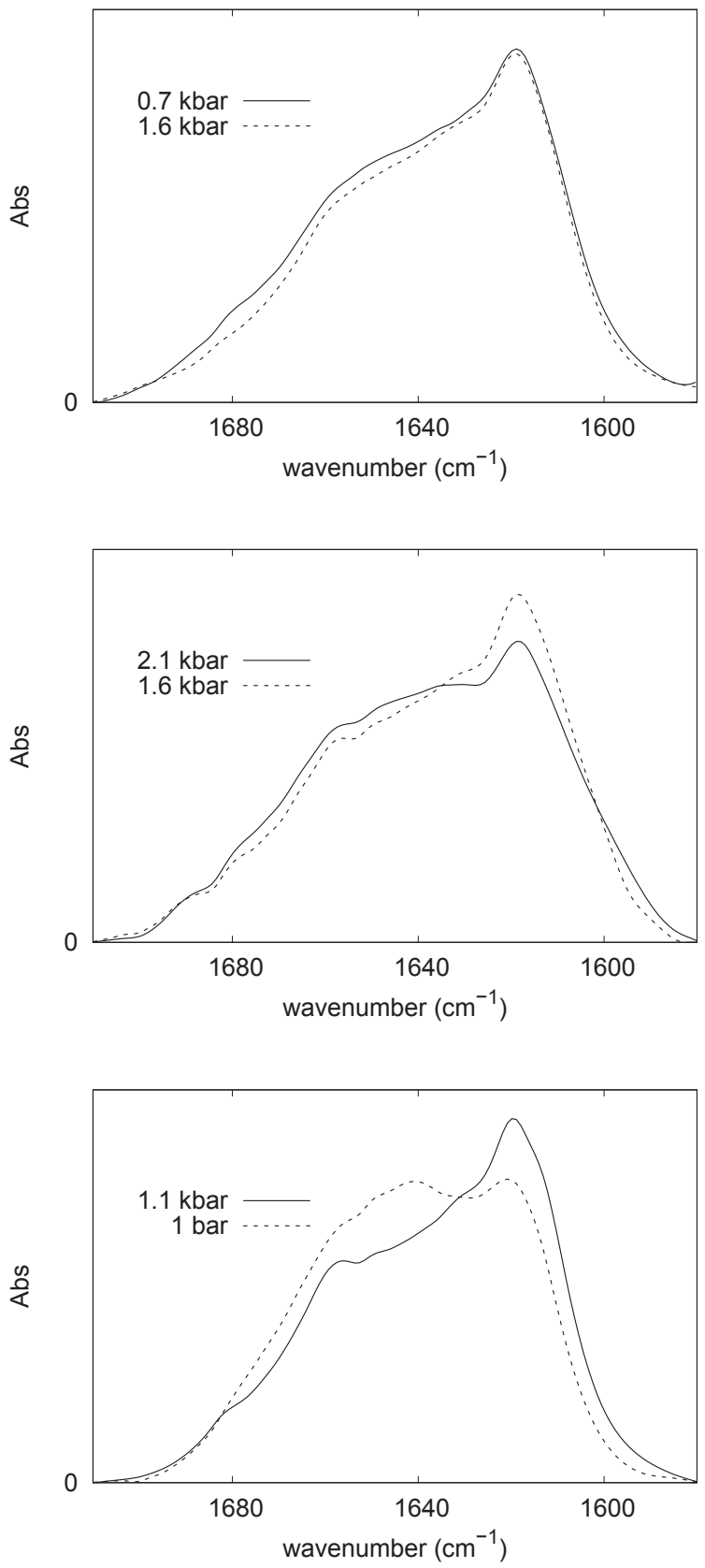

Fig. 9. Comparison between amide $\mathrm{I}^{\prime}$ absorption band of WT, A30P and A53T aSN fibrils before (initial) and after (decompressed) the first high pressure cycle. The $\beta$-sheet signal observed for A30P mutant at decompression, instead of signalling an enhanced aggregation propensity of the sample after the pressure cycle, should be ascribed to a lower pressure measured after decompression with respect to the initial one.

differences in the reversibility of the process between WT and A30P, in respect to A53T mutant, evokes the presence of a A53T stable intermediate induced by high pressure treatment, which seems to be absent in the other fibrils species. Secondly, the reversibility of high pressure fibril dissociation could be considered to be in agreement with the theory on the fibrillation phenomena [71], which considers the aggregation process not linear and claims for the presence of both primary and secondary nucleation events. This finding supports the hypothesis that small protein aggregates, the species that pass in and out of the amyloid fibrils, can be present in solution, at least in a small molar fraction. Hence, even at the highest investigated pressures, a critical number of oligomeric 


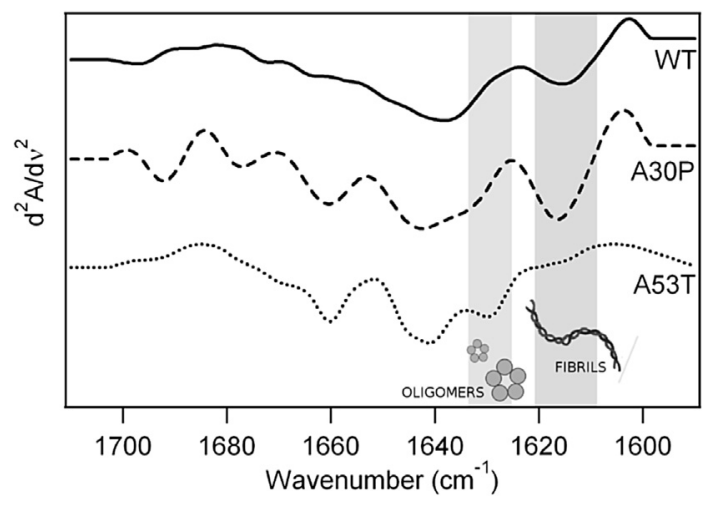

Fig. 10. $2^{\text {nd }}$-derivative Amide $\mathrm{I}^{\prime}$ equilibrium spectra of WT, A30P and A53T fibrils at high pressure recorded at the end of kinetics evolution reported in Fig. 8 (filled symbols).

species lasts in solution and with the pressure decrease these species result to be able to trigger again the amyloid aggregation.

Finally, we stress that although high hydrostatic pressure effects on fibrils dissociation cannot provide direct information on effective strategies against PD, essential knowledge of the molecular mechanisms at the basis of fibrils dissociation can be obtained from this kind of experiments. We believe these findings related to the prospect to determine alternative agents able to modify fibrils structures, as well as high hydrostatic pressure does, could be quite useful to future therapeutic perspectives.

\section{Acknowledgments}

The authors thank Elettra Sincrotrone Trieste (Grant number 20100354) because supported them as Italian funded users. M.G.O. thanks for her support the Italian grant FIRB "Future in research" RBFR12SIPT MIND.

\section{References}

[1] H. Dyson, P. Wright, Intrinsically unstructured proteins and their functions, Nat. Rev. Mol. Cell Biol. 6 (2005) 197-208.

[2] G.M. Moriarty, M.K. Janowska, L. Kang, J. Baum, Exploring the accessible conformations of n-terminal acetylated $\alpha$-synuclein, FEBS Lett. 587 (8) (2013) 1128-1138.

[3] M.D. Tuttle, G. Comellas, A.J. Nieuwkoop, D.J. Covell, D.A. Berthold, K.D. Kloepper, J.M. Courtney, J.K. Kim, A.M. Barclay, A. Kendall, W. Wan, G. Stubbs, C.D. Schwieters, V.M.Y. Lee, J.M. George, C.M. Rienstra, Solid-state nmr structure of a pathogenic fibril of full-length human $\alpha$-synuclein, Nat. Struct. Mol. Biol. 23 (2016) 409-415.

[4] P.K. Auluck, G. Caraveo, S. Lindquist, $\alpha$-synuclein: membrane interactions and toxicity in parkinson's disease, Annu. Rev. Cell Dev. Biol. 26 (2010) 211-233.

[5] V.N. Uversky, A protein-chameleon: conformational plasticity of $\alpha$-synuclein, a disordered protein involved in neurodegenerative disorders, J. Biomol. Struct. Dyn. 21 (2) (2003) 211-234.

[6] L. Breydo, J.W. Wu, V.N. Uversky, $\alpha$-synuclein misfolding and parkinson's disease, Biochim. Biophys. Acta 1822 (2012) 261-285.

[7] N. Plotegher, E. Greggio, M. Bisaglia, L. Bubacco, Biophysical groundwork as a hinge to unravel the biology of $\alpha$-synuclein aggregation and toxicity, Quat. Rev. Biophys. (2014).

[8] A. van Maarschalkerweerd, V. Vetri, A. Langkilde, V. Foderá, B. Vestergaard, Protein/lipid coaggregates are formed during $\alpha$-synuclein-induced disruption of lipid bilayers, Biomacromolecules 15 (2014) 3643-3654.

[9] M.N. Pedersen, V. Foderá, I. Horvath, A. van Maarschalkerweerd, K.N. Toft, C. Weise, F. Almqvist, M. Wolf-Watz, P. Wittung-Stafshede, B. Vestergaard, Direct correlation between ligand-induced $\alpha$-synuclein oligomers and amyloid-like fibril growth, Sci. Rep. 5 (2015) 10422.

[10] B. Winner, R. Jappelli, S.K. Maji, P.A. Desplats, L. Boyer, S. Aigner, C. Hetzer, In vivo demonstration that $\alpha$-synuclein oligomers are toxic, Proc. Natl. Acad. Sci. U. S. A. 108 (2011) 4194-4199.

[11] L. Goldschmidta, P.K. Tenga, R. Riekb, D. Eisenberg, Identifying the amylome, proteins capable of forming amyloid-like fibrils, PNAS 107 (2010) 3487-3492.

[12] J. Schnabel, The dark side of proteins, Nature 464 (2010) 828-829.

[13] E. Hazy, M. Bokor, L. Kalmar, A. Gelencser, P. Kamasa, K. Han, K. Tompa,
P. Tompa, Distinct hydration properties of wild-type and familial point mutant a53t of $\alpha$-synuclein associated with parkinson's disease, Biophys. J. 101 (2011) 2260-2266.

[14] K. Conway, J. Harper, P.T. Lansbury, Fibrils formed in vitro from $\alpha$-synuclein and two mutant forms linked to parkinson's disease are typical amyloid, Biochemistry 39 (2000) 2552-2563.

[15] G. Comellas, L.R. Lemkau, A.J. Nieuwkoop, K.D. Kloepper, D.T. Ladror, R. Ebisu, W.S. Woods, A.S. Lipton, J.M. George, C.M. Rienstra, Structured regions of $\alpha-$ synuclein fibrils include the early-onset parkinson's disease mutation sites, J. Mol. Biol. 411 (4) (2011) 881-895.

[16] L.R. Lemkau, G. Comellas, S.W. Lee, L.K. Rikardsen, W.S. Woods, J.M. George, C.M. Rienstra, Site-specific perturbations of alpha-synuclein fibril structure by the parkinson's disease associated mutations a53t and e46k, PLoS One 8 (3) (2013) e49750.

[17] M.K. Cho, H.Y. Kim, C.O. Fernandez, S. Becker, M. Zweckstetter, Conserved core of amyloid fibrils of wild type and a30p mutant $\alpha$-synuclein, Protein Sci. 20 (2) (2011) 387-395.

[18] J. Li, V.M. Uverski, A.L. Fink, Effect of familial parkinson's disease point mutations a30p and a53t on the structural properties, aggregation, and fibrillation of human $\alpha$-synuclein, Biochemistry 40 (38) (2001) 11604-11613.

[19] S. Sahay, A. Anoop, G. Krishnamoorthy, S.K. Maji, Site-specific fluorescence dynamics of $\alpha$-synuclein fibrils using time-resolved fluorescence studies: effect of familial parkinson's disease-associated mutations, Biochemistry 53 (5) (2014) 807-809.

[20] D. Foguel, M.C. Suarez, A.D. Ferrão-Gonzales, T.C.R. Porto, L. Palmieri, C.M. Einsiedler, L.R. Andrade, H.A. LAshuel, P.T. Lansbury, J.W. Kelly, J.L. Silva, Dissociation of amyloid fibrils of $\alpha$-synuclein and transthyretin by pressure reveals their reversible nature and the formation of water-excluded cavities, Proc. Natl. Acad. Sci. U. S. A. 100 (17) (2003) 9831-9836.

[21] G. de Oliveira, M.A. Marques, C. Cruzeiro-Silva, Y. Cordeiro, C. Schuabb, A. Moraes, R. Winter, H. Oschkinat, D. Foguel, M. Freitas, J. Silva, Structural basis for the dissociation of $\alpha$-synuclein fibrils triggered by pressure perturbation of the hydrophobic core, Sci. Rep. 6 (2016) 37990.

[22] R. John, J.F. Carpenter, T. Randolph, High pressure fosters protein refolding from aggregates at high concentrations, PNAS 96 (1999) 13029-13033.

[23] M.G. Ortore, F. Spinozzi, P. Mariani, A. Paciaroni, L. Barbosa, H. Amenitsch, M. Steinhart, J. Ollivier, D. Russo, Combining structure and dynamics: nondenaturing high-pressure effect on lysozyme in solution, J. R. Soc. Interface 6 (2009) S619-S634.

[24] K. Akasaka, R. Kitahara, Y.O. Kamatari, Exploring the folding energy landscape with pressure, Arch. Biochem. Biophys. 531 (2013) 110-115. Protein Folding and Stability.

[25] D. Russo, M.G. Ortore, F. Spinozzi, P. Mariani, C. Loupiac, B. Annighofer A. Paciaroni, The impact of high hydrostatic pressure on structure and dynamics of beta-lactoglobulin, Biochim. et Biophys. Acta (BBA)-Gen. Subj. 1830 (10) (2013) 4974-4980.

[26] E. Rosenbaum, F. Gabel, M.A. Dura, S. Finet, C. Clery-Barraud, P. Masson, B. Franzetti, Effects of hydrostatic pressure on the quaternary structure and enzymatic activity of a large peptidase complex from Pyrococcus horikoshii, Arch. Biochem. Biophys. 517 (2012) 104-110.

[27] M.G. Ortore, F. Spinozzi, C. Carsughi, P. Mariani, M. Bonetti, G. Onori, High pressure small-angle neutron scattering study of the aggregation state of $\beta$ lactoglobulin in water and water/ethylene glycol solutions, Chem. Phys. Lett. 418 (2005) 338-342.

[28] S. Mangialardo, F. Piccirilli, A. Perucchi, P. Dore, P. Postorino, Raman analysis of insulin denaturation induced by high-pressure and thermal treatments, J. Raman Spectrosc. 43 (2012) 692-700.

[29] F. Piccirilli, S. Mangialardo, P. Postorino, L. Baldassarre, S. Lupi, A. Perucchi, Sequential dissociation of insulin amyloids probed by high pressure fourier transform infrared spectroscopy, Soft Matter (2012) 11863-11870.

[30] F. Piccirilli, S. Mangialardo, P. Postorino, S. Lupi, A. Perucchi, Infrared microspectroscopy study of insulin crystals at high pressure, J. Phys. Conf. Ser. 359 (2012) 012014.

[31] F. Piccirilli, S. Mangialardo, P. Postorino, S. Lupi, A. Perucchi, Ftir analysis of the high pressure response of native insulin assemblies, J. Mol. Struct. 1050 (2013) 159-165.

[32] J. Roche, J.A. Caro, D.R. Norberto, P. Barthe, C. Roumestand, J.L. Schlessmand, A.E. Garcia, B. Garca-Moreno, C.A. Royer, Cavities determine the pressure unfolding of proteins, Proc. Natl. Acad. Sci. U. S. A. 109 (18) (2012) 6945-6950.

[33] J. Silva, D. Foguel, C. Royer, Pressure provides new insights into protein folding, dynamics and structure, Trends Biochem. Sci. 26 (2001) 612-618.

[34] F. Meersman, C.M. Dobson, Probing the pressure-temperature stability of amyloid fibrils provides new insights into their molecular properties, Biochim. Biophys. Acta (BBA) - Proteins Proteom. 1764 (2006) 452-460.

[35] L. Tosatto, A.O. Andrighetti, N. Plotegher, V. Antonini, I. Tessari, L. Ricci L. Bubacco, M.D. Serra, $\alpha$-synuclein pore forming activity upon membrane association, Biochim. Biophys. Acta (BBA) - Biomembr. 1818 (2012) 2876-2883.

[36] M.E. van Raaij, J. van Gestel, I.M.J. Segers-Nolten, S.W. de Leeuw, V. Subramaniam, Concentration dependence of $\alpha$-synuclein fibril length assessed by quantitative atomic force microscopy and statistical-mechanical theory, Biophys. J. 95 (10) (2008) 4871-4878.

[37] K. Pressl, M. Kriechbaum, M. Steinhart, P. Laggner, High pressure cell for small- and wide-angle x-ray scattering, Rev. Sci. Instrum. 68 (1997) 4588-4592. 
[38] H. Mao, J. Xu, P. Bell, Calibration of the ruby pressure gauge to 800 kbar under quasi-hydrostatic conditions, J. Geophys. Res. 91 (1987) 4673-4676.

[39] M. Bisaglia, S. Mammi, L. Bubacco, Structural insights on physiological functions and pathological effects of $\alpha$-synuclein, FASEB J. 23 (2009) 329-340.

[40] J.S. Pedersen, P. Schurtenberger, Scattering functions of semiflexible polymers with and without excluded volume effects, Macromolecules 29 (1996) $7602-7612$

[41] F. Spinozzi, C. Ferrero, M.G. Ortore, A. De Maria Antolinos, P. Mariani, GENFIT: software for the analysis of small-angle X-ray and neutron scattering data of macromolecules in-solution, J. App. Cryst. 47 (2014) 1132-1139.

[42] R. Roychaudhurl, M. Yang, M.M. Hoshi, D.B. Teplow, Amyloid $\beta$-protein assembly and alzheimer disease, J. Biol. Chem. 284 (8) (2008) 4749-4753.

[43] C.L. Heldt, S. Zhang, G. Belfort, Asymmetric amyloid fibril elongation: A new perspective on a symmetric world, Proteins 79 (2008) 92-98.

[44] R. Carrotta, J. Barthes, A. Longo, V. Martorana, M. Manno, G. Portale P.L.S. Biagio, Large size fibrillar bundles of the alzheimer amyloid $\beta$-protein, Eur. Biophys. J. 36 (2007) 701-709.

[45] D.E. Otzen, Amyloid Fibrils and Prefibrillar Aggregates: Molecular and Biological Properties, Wiley-VCH, 2013.

[46] C. Ricci, F. Spinozzi, P. Mariani, M.G. Ortore, Protein amyloidogenesis investigated by small angle scattering, Curr. Pharm. Des. 22 (2016) 3937-3949.

[47] C.C. Curtain, N.M. Kirby, H.D.T. Mertens, K.J. Barnham, R.B. Knott, C.L. Masters R. Cappai, A. Rekas, V.B. Kenche, T. Ryan, Alpha-synuclein oligomers and fibrils originate in two distinct conformer pools: a small angle x-ray scattering and ensemble optimisation modelling study, Mol. Biosyst. 11 (2015) 190-196.

[48] S.B. Nielsen, F. Macchi, S. Raccosta, A.E. Langkilde, L. Giehm, A. Kyrsting A.S.P. Svane, M. Manno, G. Christiansen, N.C. Nielsen, L. Oddershede, B. Vestergaard, D.E. Otzen, Wildtype and a30p mutant alpha-synuclein form different fibril structures, PLoS One 8 (7) (2013) e67713.

[49] V.I. Petrenko, M.V. Avdeev, V.M. Garamus, M. Kubovcikova, Z. Gazov, K. Siposov, L.A. Bulavin, L. Almásy, V.L. Aksenov, P. Kopcansky, Structure of amyloid aggregates of lysozyme from small angle x-ray scattering data, Phys. Solid State 56 (1) (2014) 129-133.

[50] M.E. van Raaij, I.M. Segers-Nolten, V. Subramaniam, Quantitative morphological analysis reveals ultrastructural diversity of amyloid fibrils from $\alpha$ synuclein mutants, Biophys. J. 91 (11) (2006) 96-98.

[51] M. Vilar, H.T. Chou, T, Luhrs, S.K. Maji, D. Riek-Loher, R. Verel, G, Manning, H. Stahlberg, R. Riek, The fold of $\alpha$-synuclein fibrils, Proc. Natl. Acad. Sci. U. S. A. 105 (2008) 8637-8642.

[52] L. Giehm, D.I. Svergun, D.E. Otzen, B. Vestergaard, Low-resolution structure of a vesicle disrupting $\alpha$-synuclein oligomer that accumulates during fibrillation, Proc. Natl. Acad. Sci. U. S. A. 108 (2011) 3246-3251.

[53] S.H. Chen, Small angle neutron scattering studies of the structure and interaction in micellar and microemulsion systems, Annu. Rev. Phys. Chem. 37 (1986) 351-399.

[54] M.R. Mangione, A. Palumbo Piccionello, C. Marino, M.G. Ortore, P. Picone, S. Vilasi, M. Di Carlo, S. Buscemi, D. Bulone, P.L. San Biagio, Photo-inhibition of a $\beta$ fibrillation mediated by a newly designed fluorinated oxadiazole, RSC Adv. 5 (2015) 16540-16548.

[55] W. Paslawski, M. Andreasen, S.B. Nielsen, N. Lorenzen, K. Thomsen, J.D. Kaspersen, J.S. Pedersen, D.E. Otzen, High stability and cooperative unfolding of $\alpha$-synuclein oligomers, Biochemistry 53 (2014) 6252-6263.

[56] F. Spinozzi, P. Mariani, I. Mičetić, C. Ferrero, D. Pontoni, M. Beltramini, Quaternary structure heterogeneity of oligomeric proteins: a saxs and sans study of the dissociation products of octopus vulgaris hemocyanin, PLoS One 7 (2012) e49644.

[57] A. Guinier, G. Fournet, Small Angle Scattering of X-ray, Wiley, New York, 1955.

[58] J. Macdonald, Review of some experimental and analytical equations of state, Rev. Mod. Phys. 41 (1969) 316-349.

[59] L. Paccamiccio, M. Pisani, F. Spinozzi, C. Ferrero, S. Finet, P. Mariani, Pressure effects on lipidic direct phases: the dodecyl trimethyl ammonium chloridewater system, J. Phys. Chem. B 110 (2006) 12410-12418.

[60] D.P. Kharakoz, Protein compressibility, dynamics, and pressure, Biophys. J. 79 (1) (2000) 511-525.

[61] M.G. Ortore, F. Spinozzi, S. Vilasi, I. Sirangelo, G. Irace, A. Shukla, T. Narayanan, R. Sinibaldi, P. Mariani, Time-resolved small-angle X-ray scattering study of the early stage of amyloid formation of an apomyoglobin mutant, Phys. Rev. E 84 (2011) 061904.

[62] N. Lorenzen, S.B. Nielsen, A.K. Buell, J.D. Kaspersen, P. Arosio, B.S. Vad, W. Paslawski, G. Christiansen, Z. Valnickova-Hansen, M. Andreasen, JJ. Enghild, J.S. Pedersen, C.M. Dobson, T.P.J. Knowles, D.E. Otzen, The role of stable $\alpha$-synuclein oligomers in the molecular events underlying amyloid formation, JACS 136 (2014) 3859-3868.

[63] J.L. Silva, A.C. Oliveira, T.C.R.G. Vieira, G.A.P. de Oliveira, M.C. Suarez, D. Foguel, High-pressure chemical biology and biotechnology, Chem. Rev. 114 (2014) 7239-7267.

[64] J.M. Chalmers, P.R. Griffiths, Handbook of Vibrational Spectroscopy, Wiley and Sons, 1986.

[65] V. Militello, C. Casarino, A. Emanuele, A. giostra, F. Pullara, M. Leone, Aggregation kinetics of bovine serum albumin studied by ftir spectroscopy and light scattering, Biophys. Chem. 107 (2004) 175-187.

[66] A. Dong, P. Huang, W. Caughey, Protein secondary structures in water from second-derivative amide i infrared spectra, Biochemistry 29 (1990) 3303-3308.

[67] V.N. Uversky, J. Lie, P. Souillac, I.S. Millett, S. Doniach, R. Jakes, M. Goedert, A.L. Fink, Biophysical properties of the synucleins and their propensities to fibrillate, J. Biol. Chem. 277 (14) (2002) 11970-11978.

[68] B.R. Shah, A. Maeno, H. Matsuo, H. Tachibana, K. Akasaka, Pressure-accelerated dissociation of amyloid fibrils in wild-type hen lysozyme, Biophys. J. 102 (2012) 121-126

[69] P. Mandal, N. Eremina, A. Barth, Formation of two different types of oligomers in the early phase of ph-induced aggregation of the alzheimer a $\beta(12-28)$ peptide, J. Phys. Chem. B 116 (41) (2012) 12389-12397.

[70] K. Hasegawa, K. Ono, M. Yamada, H. Naiki, Kinetic modeling and determination of reaction constants of alzheimer's $\beta$-amyloid fibril extension and dissociation using surface plasmon resonance, Biochemistry 41 (2002) 13489-13498. PMID: 12427009.

[71] S.I.A. Cohen, S. Linse, L.M. Luheshi, E. Hellstrand, D.A. White, L. Rajah, D.E. Otzen, M. Vendruscolo, C.M. Dobson, T.P.J. Knowles, Proliferation of amyloid- $\beta 42$ aggregates occurs through a secondary nucleation mechanism, Proc. Natl. Acad. Sci. U. S. A. 110 (2013) 9758-9763. 